\title{
Selecting a Mix of Prevention Strategies against Cervical Cancer for Maximum Efficiency with an Optimization Program
}

\author{
Nadia Demarteau, ${ }^{1}$ Thomas Breuer ${ }^{2}$ and Baudouin Standaert ${ }^{1}$ \\ 1 Health Economics, Global Vaccine Development, GlaxoSmithKline Biologicals, Wavre, Belgium \\ 2 Global Vaccine Development, GlaxoSmithKline Biologicals, Wavre, Belgium
}

\section{Abstract}

Background: Screening and vaccination against human papillomavirus (HPV) can protect against cervical cancer. Neither alone can provide $100 \%$ protection. Consequently it raises the important question about the most efficient combination of screening at specified time intervals and vaccination to prevent cervical cancer.

Objective: Our objective was to identify the mix of cervical cancer prevention strategies (screening and/or vaccination against HPV) that achieves maximum reduction in cancer cases within a fixed budget.

Methods: We assessed the optimal mix of strategies for the prevention of cervical cancer using an optimization program. The evaluation used two models. One was a Markov cohort model used as the evaluation model to estimate the costs and outcomes of 52 different prevention strategies. The other was an optimization model in which the results of each prevention strategy of the previous model were entered as input data. The latter model determined the combination of the different prevention options to minimize cervical cancer under budget, screening coverage and vaccination coverage constraints.

We applied the model in two countries with different healthcare organizations, epidemiology, screening practices, resource settings and treatment costs: the UK and Brazil. 100000 women aged 12 years and above across the whole population over a 1-year period at steady state were included.

The intervention was papanicolaou (Pap) smear screening programmes and/or vaccination against HPV with the bivalent HPV 16/18 vaccine (Cervarix ${ }^{\circledR}[$ Cervarix is a registered trademark of the GlaxoSmithKline group of companies]). The main outcome measures were optimal distribution of the population between different interventions (screening, vaccination, screening plus vaccination and no screening or vaccination) with the resulting number of cervical cancer and associated costs.

Results: In the base-case analysis (= same budget as today), the optimal prevention strategy would be, after introducing vaccination with a coverage rate of $80 \%$ in girls aged 12 years and retaining screening coverage at 
pre-vaccination levels (65\% in the UK, $50 \%$ in Brazil), to increase the screening interval to 6 years (from 3 ) in the UK and to 5 years (from 3 ) in Brazil. This would result in a reduction of cervical cancer by $41 \%$ in the UK and by $54 \%$ in Brazil from pre-vaccination levels with no budget increase. Sensitivity analysis shows that vaccination alone at $80 \%$ coverage with no screening would achieve a cervical cancer reduction rate of $20 \%$ in the UK and $43 \%$ in Brazil compared with the pre-vaccination situation with a budget reduction of $30 \%$ and $14 \%$, respectively. In both countries, the sharp reduction in cervical cancer is seen when the vaccine coverage rate exceeds the maximum screening coverage rate, or when screening coverage rate exceeds the maximum vaccine coverage rate, while maintaining the budget. As with any model, there are limitations to the value of predictions depending upon the assumptions made in each model.

Conclusions: Spending the same budget that was used for screening and treatment of cervical cancer in the pre-vaccination era, results of the optimization program show that it would be possible to substantially reduce the number of cases by implementing an optimal combination of HPV vaccination ( $80 \%$ coverage) and screening at pre-vaccination coverage $(65 \% \mathrm{UK}$, $50 \%$ Brazil) while extending the screening interval to every 6 years in the UK and 5 years in Brazil.

\section{Key points for decision makers:}

- Two cervical cancer (CC) prevention strategies exist: secondary (screening) and primary (HPV vaccination)

- Optimization models can be used to determine the optimal mix of primary and secondary prevention strategies minimizing $\mathrm{CC}$ burden under budget and logistic constraints

- Extending existing screening intervals while implementing vaccination in both the UK and Brazil could result in a substantial CC reduction while maintaining the current budget

\section{Introduction}

Cervical cancer is the second most common cancer in women aged under 35 years in the UK, ${ }^{[1]}$ with an estimated 529000 cases and 274000 deaths per year worldwide. ${ }^{[2]}$ Different strategies are available for the prevention of cervical cancer, including secondary prevention using screening and the treatment of pre-cancerous lesions and primary prevention using vaccination against human papillomavirus (HPV).

Cytology-based screening programmes have contributed to a decrease of up to $80 \%$ in the in- cidence and the mortality of cervical cancer in countries with a well established, organized screening programme such as in the Netherlands, the UK and Finland. ${ }^{[3,4]}$ However, cytology-based screening programmes do not achieve full coverage in any country. Together with factors such as sensitivity of the screening method, treatment failure and the level of resources required for an adequate follow-up of patients, screening has sometimes a limited impact. Furthermore, it seems difficult to implement cytology-based screening programmes in low-resource settings. So this prevention strategy fails particularly in the developing world 
where the disease is most prevalent. ${ }^{[5]}$ Infection with HPV is a necessary condition for the development of cervical cancer, ${ }^{[6,7]}$ opening up the possibility of primary prevention with HPV vaccination. Eight HPV genotypes (HPV 16, 18, 45, $31,33,52,58$ and 35) account for more than $90 \%$ of cervical cancer cases, ${ }^{[8]}$ and HPV 16 and 18 account for around $70 \% .{ }^{[9]}$ Two HPV vaccines are currently available, a bivalent HPV 16/18 AS04adjuvanted vaccine and a quadrivalent HPV 6/11/ 16/18 L1 virus-like particle vaccine that covers two non-oncogenic HPV types (HPV 6 and 11) as well as the oncogenic types HPV 16 and 18. Both vaccines have an efficacy of around $98 \%$ against the cervical cancer vaccine HPV types (HPV 16 and 18), ${ }^{[10-16]}$ but with a different cross-protection profile against oncogenic non-vaccine HPV-types. ${ }^{[17,18]}$ The implementation of vaccination varies widely between countries with regard to the strategy selection (national immunization programme or individual based); the logistics (via a separately established vaccination setting or via primary healthcare); the age group targeted; and the gender selection (female only or both). Published vaccine coverage rates range from more than $70 \%$ in the UK to around $25 \%$ in girls aged 12 years in the US. ${ }^{[19-21]}$

Numerous studies have investigated the cost effectiveness of vaccination, ${ }^{[22-25]}$ and most have concluded that vaccination in addition to existing screening programmes should provide significant incremental health benefit so that vaccination is cost effective at the recommended vaccine price in the developed world. ${ }^{[23,24]}$ However, neither vaccination nor screening alone can provide $100 \%$ protection against cervical cancer, so an optimal prevention strategy should be a combination of both. This raises the important question about what is the most efficient combination strategy of screening and vaccination to prevent cervical cancer.

Incremental cost-effectiveness analyses have limitations in evaluating multiple combinations of different interventions in relation to a budget and other constraints. ${ }^{[26-30]}$ Standard cost-effectiveness analysis does not take into account any affordability constraint. It is therefore not well suited to the problem of finding the optimal mix of several complementary interventions to provide the most efficient use of healthcare resources. Similarly, although budget impact analysis provides a measure of affordability, that type of analysis only presents cost over time and is not linked to any achievement of specific outcome measures. We present here an alternative economic evaluation tool - optimization modelling - that applies linear programming to select the combination of interventions that achieves the best outcome while fulfilling the constraints introduced in the model, such as no increase compared with the prevaccination budget. ${ }^{[31-37]}$ Linear programming is a mathematical method for determining the input values that give the best outcome (such as maximum profit or lowest cost) for a list of requirements expressed as linear equations. ${ }^{[38]}$ The technique is not new. It has been applied in many different areas such as transport, agriculture, industry and banking ${ }^{[39]}$ before it recently reached the medical community and has been applied to a few health and healthcare situations. ${ }^{[32-37]}$ To demonstrate that this approach is generally applicable across different healthcare systems, we applied the model in two countries with different healthcare organizations, epidemiology, screening practices, resource settings and treatment costs: one in Europe (the UK), and one in Latin America (Brazil). Also, a cost-effectiveness model for HPV vaccination and screening was already developed and published for both countries. ${ }^{[40,41]}$

\section{Methods}

Development of an optimization program for cervical cancer prevention requires the use of two different models, an 'evaluation' model and an 'optimization' model. The evaluation model separately assesses multiple prevention scenarios (screening; vaccination; screening plus vaccination; and no screening or vaccination) to produce outcome measures for each prevention scenario tested. One outcome is the total cost from a healthcare provider's perspective, including the costs of screening; of treatment of pre-cancerous lesions and cervical cancer; and of vaccination. The other is a health outcome result, i.e. the incident number of cervical cancer cases. Cervical cancer was selected as the health outcome measure because it represents 


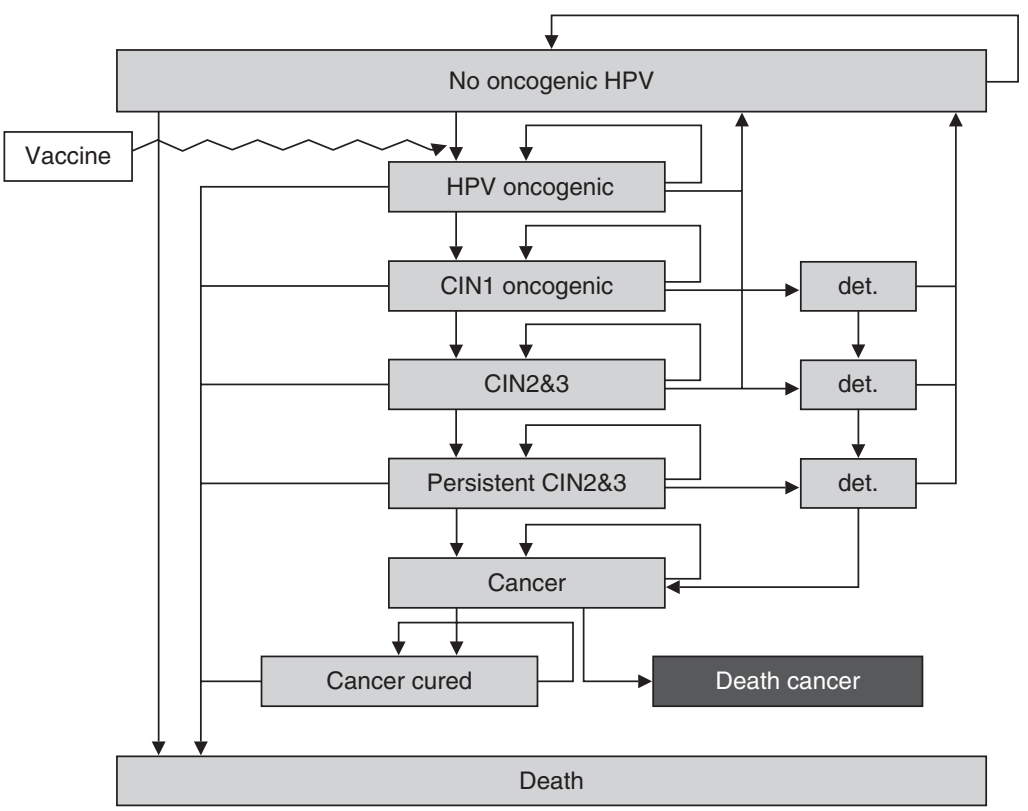

Fig. 1. Structure of the evaluation model. Reproduced from Debicki et al., ${ }^{[40]}$ with permission from Elsevier. $\mathrm{CIN}=$ cervical intraepithelial neoplasia; det. = subject with disease detected through screening; $\mathbf{H P V}=$ human papillomavirus.

the ultimate goal of cervical cancer prevention strategies; other options such as QALYs gained could have been considered. Subsequently, the results of the evaluation model were then used as input data in the optimization model. Applying linear programming, this optimization model determined the optimal distribution of the population between the different screening and vaccination strategies under specific constraints of budget, and screening- and vaccination coverage. The purpose of these constraints is to approximate to a real-life setting where decisions are to be taken within a limited budget and $100 \%$ vaccination and screening coverage can never be achieved.

\section{Evaluation Model}

The evaluation model used was a Markov cohort model built in Microsoft ${ }^{\circledR}$ Excel that assesses the clinical and economic effect of vaccination and cytology-based screening compared with cytology-based screening alone. The cohort model estimates the cost and the number of cervical cancer cases for a population of 100000 women at pre- vention steady state level, for each prevention strategy analysed separately. Assuming that the population demographics are stable over time, it is then possible to assess the lifetime cohort results as if the results were obtained across a whole population over a 1-year period only. Under these conditions, each cycle of the Markov cohort model running over a lifetime period represents one age group of the cross-sectional whole population demographic pyramid in a 1-year period.

The Markov cohort model consists of 12 health states, shown schematically in figure 1. It uses a Markov process with 1-year cycle length to describe the natural history of cervical cancer disease.

Adaptation of the model to the UK and the Brazilian settings were previously developed and published elsewhere. Precise information on the model input data and outcomes are available in these publications. ${ }^{[40,41]}$ The analysis was performed from a healthcare provider's perspective, so only direct medical costs were included. The cost of the vaccine was set at $£ 80$ per dose in the UK based on the current list price and \$US30 per dose in Brazil based on the price in a neighbouring country in 2010 
(Panama). As the estimated model results were then used in a cross-sectional 1-year assessment of a whole population, no discount rate was applied.

The different prevention strategies considered were screening; vaccination; screening plus vaccination; and no screening or vaccination, representing the options currently available for cervical cancer prevention in those countries. Only cytology-based screening was included in the model. Cervical cancer screening covered women aged from 25 to 65 years in the UK and from 18 to 65 years in Brazil, following the age range currently covered in each country. The screening intervals were varied from every year to every 25 years (i.e. women are then screened only twice over their lifetime) with a 1-year increment between each scenario. It was assumed that vaccination induces lifelong protection and targets 12-year-old girls. Vaccine efficacy was taken as $98 \%$ against the vaccine HPV-types 16 and 18, and $68.3 \%$ against the ten most frequent non-vaccine HPV-types in cervical cancer, reflecting the efficacy profile of the bivalent vaccine in an HPV-naïve population. ${ }^{[10]}$ Overall vaccine efficacy was calculated from the proportion of each HPV type in cervical cancer ${ }^{[42]}$ as previously described. ${ }^{[40,41]}$ The detailed values included to calculate the efficacy are reported in table I. In total, 52 different strategies were tested for each country.

The model was run with a cohort of girls over their lifetime for each scenario separately. The results of each scenario were used to estimate the number of cervical cancer cases and total costs expected over 1 year at steady state by dividing those lifetime results by the total number of lifeyears lived by the cohort. The latter was then mul- tiplied by 100000 to estimate the projected values at steady state for each strategy per 100000 women. The estimated cervical cancer cases and costs of each prevention strategy were then used as input variables in the optimization model.

\section{Optimization Model}

The optimization model was also developed in Microsoft Excel and uses Solver of Microsoft Excel with linear programming. The model distributes the population between the 52 pre-defined prevention strategies to achieve the subject function linked with several constraints. The different 52 strategies are therefore mutually exclusive. The model can be used to analyse questions such as the optimal mix of cervical cancer prevention strategies to minimize the expected cervical cancer incidence rate within a fixed budget, with additional constraints on screening and vaccination coverage. It can also estimate the minimum additional budget required to achieve a pre-defined health goal, such as a specified reduction in cervical cancer case numbers, within a pre-defined time frame using a combination of prevention strategies.

Formulation of the resource allocation problem in terms of linear programming is convenient because only minor adjustments are needed to analyse different optimization problems with different constraints. One critical condition needs to be fulfilled when using linear programming; namely that a linear incremental change in an independent variable results in a linear incremental change in the dependent output measure (i.e. if the value of an input variable is doubled for a given strategy, the value of the output measure relative to that strategy

Table I. Values included to calculate vaccine efficacy

\begin{tabular}{|c|c|c|c|}
\hline Lesion & Vaccine efficacy [\%] & Type distribution UK (WHO) [\%] & Type distribution Brazil (WHO) [\%] \\
\hline$\overline{\mathrm{CIN} 1-\mathrm{HPV} 16 / 18}$ & $98^{[10,14]}$ & 29 & 35 \\
\hline CIN1 cross-protection ${ }^{a}$ & $47.7^{[43]}$ & 64 & 63 \\
\hline CIN2/3 - HPV 16/18 & $98^{[10,14]}$ & 61 & 57 \\
\hline CIN2/3 cross-protection ${ }^{a}$ & $68.4^{[16]}$ & 39 & 24 \\
\hline Cervical cancer - HPV 16/18 & $98^{[10,14]}$ & 77 & 70 \\
\hline Cervical cancer cross-protection $^{a}$ & $68.4^{[16]}$ & 17 & 29 \\
\hline
\end{tabular}


would also double). This is the case in the current analysis.

The mathematical problem is therefore defined as follows:

A single linear objective function:

$$
\text { Minimize } \sum_{i=1}^{52} C C_{i} \times x_{i}
$$

Subject to multiple linear constraints:

$$
\sum_{i=1}^{52} b_{i} \times x_{i} \leq B
$$

(Budget constraint)

$$
0 \leq x_{i} \leq 1 \text { for } i=1, \ldots, 52
$$

(Strategy coverage bounds)

$$
\sum_{i=1}^{52} x_{i}=1
$$

(Complete population distribution)

$$
\sum_{s=1}^{50} x_{s} \leq \operatorname{Cov}_{1}
$$

(Screening coverage upper bound)

$$
\sum_{v=1}^{26} x_{v} \leq \operatorname{Cov}_{2}
$$

(Vaccination coverage upper bound)

$$
x_{\text {nprev }} \geq \text { Minimum value between } 1-\operatorname{Cov}_{1}
$$

and $1-\operatorname{Cov}_{2}$

(Upper bound on population with no prevention) with $x_{i} \in \mathrm{R}$ (a real number).

$x_{i}$ is a continuous decision variable representing the proportion of the population in the strategy $i$; these $52 x_{i}$ are the optimization variables. $i(i=$ $1, \ldots, 52)$ denotes the different pre-defined prevention strategies, of which there are 25 with screening alone (with 25 intervals from 1 to 25 years using a 1-year increment); 1 with vaccination alone; 25 with vaccination combined with screening (with screening intervals from 1 to 25 years using a 1 -year increment); and 1 with no prevention measure.

$s$ denotes a subset of the $i$ strategies corresponding to strategies involving screening (50 strategies out of 52: 25 with screening alone, 25 with both vaccination and screening).

$v$ denotes a subset of the $i$ strategies corresponding to strategies involving vaccination (26 strategies out of 52: 1 with vaccination alone and 25 with both vaccination and screening).

$x_{\text {nprev }}$ represents, among the $x_{i}$, the proportion of population with no preventive measure.

$\mathrm{Cov}_{1}$ and $\mathrm{Cov}_{2}$ denote the upper bounds for coverage in terms of screening and vaccination, respectively.

$C C_{i}$ represents the number of cervical cancer cases at steady state for 100000 women, all being under the strategy $i$ as estimated by the evaluation model.

$b_{i}$ is the total budget for 100000 women at steady state under the strategy $i$ as estimated by the evaluation model.

$B$ is the overall budget across the population (corresponding to the pre-vaccination budget in the base case).

\section{Base Case}

The base-case analysis arbitrarily assumed that the maximum screening coverage $\left(\operatorname{Cov}_{1}\right)$ is the pre-vaccination coverage rate $(65 \%$ of the women are screened in the UK and 50\% in Brazil with a screening interval of 3 years in both countries); ${ }^{[40,41,44]}$ maximum vaccination coverage $\left(\mathrm{Cov}_{2}\right)$ was set to $80 \%$; and the overall budget $(B)$ was not higher than the pre-vaccination budget allotted to screening and treatment of cervical lesions. The coverage rates are consistent with those currently observed in the UK and Brazil. In addition, if any difference exists between vaccine coverage rate and screening included in the constraint, it is assumed that people who are not vaccinated are also not screened and vice versa. It implies that a woman who is not screened is also unlikely to be vaccinated and that a woman who is not vaccinated is also unlikely to be screened. The difference between the two would only result in different coverage constraints between the two strategies. As a result of this assumption, in both countries the proportion of the population receiving no prevention $\left(x_{\text {nprev }}\right)$ is equal to the proportion remaining when the maximum permitted vaccination coverage of $80 \%$ is achieved. Where the screening interval varies by age group (e.g. in the UK, women are invited for screening every 3 years if aged 25-49 years and every 5 years if aged 50-64 years), the model 
applied a single screening interval across all age groups as an approximation.

\section{Sensitivity Analysis}

Univariate sensitivity analyses were conducted to estimate the effects on the number of cervical cancer cases, the budget and the screening interval when changing the constraint on budget, screening coverage or vaccination coverage, as well as the duration of vaccine protection. These constraints were selected because they represent the important aspects of a health prevention policy that may be subject to variation in the future. The budget constraint was varied from a $20 \%$ reduction to a $150 \%$ increase over the pre-vaccination levels, while the screening and the vaccination coverage levels were varied from $0 \%$ to $100 \%$. The sensitivity analysis on the vaccine duration assumes a 25-year duration of protection ${ }^{[45]}$ and a booster coverage of $40 \%$ that would lead to a lifetime protection. All other parameters were kept constant as in the base-case condition.

\section{Results}

\section{Base Case}

UK

The optimal allocation of resources in the UK is presented in figure $2 \mathrm{a}$, with the pre-vaccination situation shown for comparison. The stacked columns represent the proportion of women in the population receiving each intervention, shown on the left-hand axis. The individual data points (represented by a diamond and a triangle) show the screening interval for women who are vaccinated and not vaccinated, respectively, shown on the right-hand axis. Compared with the prevaccination situation in which $65 \%$ of the women are screened every 3 years, the optimal scenario would be that $65 \%$ of the women are vaccinated and also screened every 6 years, and that $15 \%$ would be vaccinated but not screened, taking the total vaccination coverage rate to its maximum level of $80 \%$. The remaining $20 \%$ would not receive any prevention. This mix of interventions would lead to a reduction of $41 \%$ in the number of cervical
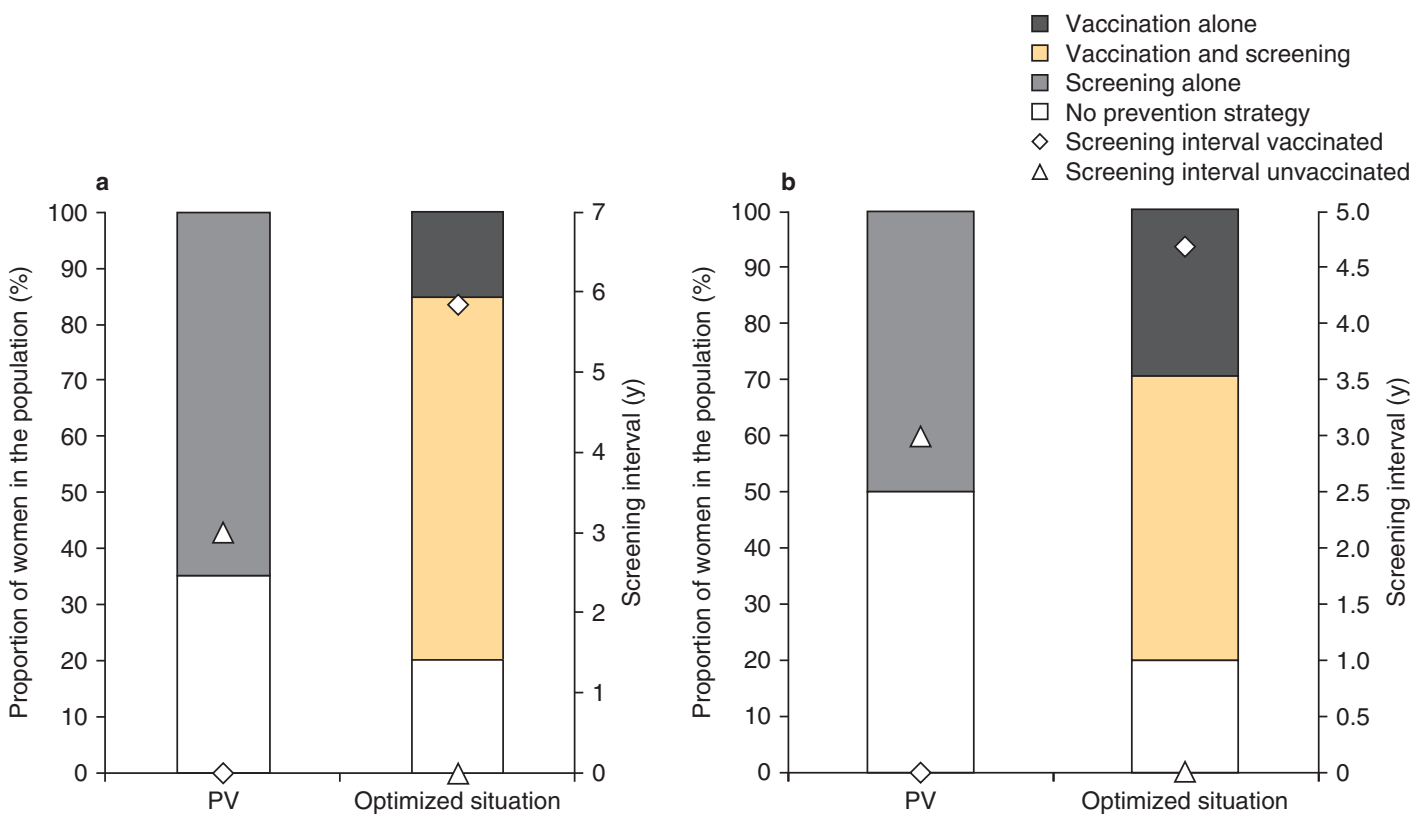

Fig. 2. Pre-vaccination strategy and base-case optimal budget allocation for (a) the UK and (b) Brazil. The left axis refers to the population distribution (bars), and the right to the screening interval ( $\Delta$ : screened only; $\diamond$ : vaccinated and screened; where $\diamond$ or $\Delta=0$ correspond to having no women under that specific scenario, e.g. screened only or screened and vaccinated). $\mathbf{P V}=$ pre-vaccination strategy. 
cancer cases from the pre-vaccination level with no budget change.

\section{Brazil}

The optimal allocation of resources in Brazil is presented in figure $2 \mathrm{~b}$, with the pre-vaccination situation shown for comparison. Compared with the pre-vaccination situation in which $50 \%$ of the women are screened every 3 years, the optimal situation would be that $50 \%$ are vaccinated and also screened every 5 years on average; $30 \%$ would be vaccinated without screening; and the remaining $20 \%$ would not receive any prevention. As in the UK base case, the optimal allocation of resources has both preventive interventions at their maximum coverage levels, $80 \%$ for vaccination and the pre-vaccination level of $50 \%$ for screening, with the additional assumption that people who are not vaccinated would also not be screened, therefore resulting in $20 \%$ receiving no prevention. This mix would result in a reduction of 54\% in the number of cervical cancer cases from the pre-vaccination level for the same budget.

\section{Sensitivity Analysis}

Sensitivity analyses considering the budget constraint, the vaccine coverage, the screening coverage and the vaccine duration are reported for both countries in figures 3-6, respectively.

In both countries, budget increases from prevaccination levels allow for a reduction in the screening interval while vaccination coverage remains at the maximum level of $80 \%$ throughout (figure $3 \mathrm{a}$ and $\mathrm{b}$ ). Shortening the screening interval brings a further decrease in cervical cancer cases with increasing budget, but the reduction is marginal and tends to reach an early plateau (figure $3 \mathrm{c}$ ). The total budget in Brazil reaches a plateau at a level that is $60 \%$ higher than baseline, because the screening interval has reached its maximum frequency (yearly) and both screening and vaccination have reached the maximum coverage rates permitted under the model constraints (figure $3 \mathrm{~d}$ ). A budget reduction of $10 \%$ or $20 \%$ results in an extended screening interval (above 10 years) combined with a maximum vaccine coverage rate of $80 \%$ in both countries (figure $3 \mathrm{a}$ and $b$ ). With the scenarios of a reduced budget, optimal allocation as in figure $3 \mathrm{a}$ and $\mathrm{b}$ reduces the number of cervical cancer cases by at least $30 \%$ compared with the pre-vaccination situations (figure $3 \mathrm{c}$ ).

Figure 4 shows the effect of varying the vaccination coverage over the full range from $0 \%$ to $100 \%$. In both countries, the number of cervical cancer cases shows a substantial decline when the vaccine coverage becomes higher than the prevaccination screening coverage of $65 \%$ in the UK and $50 \%$ in Brazil (figure 4c). In both countries, the optimal screening interval in women who are vaccinated and screened is longer than the screening interval in women who receive screening alone (figure $4 \mathrm{a}$ and $\mathrm{b}$ ); note that screening alone is an option only when the vaccination coverage allowed is lower than the screening coverage. In the UK, the screening interval does not change much with increasing vaccine coverage (figure $4 a$ ), whereas in Brazil the screening interval in women who are both screened and vaccinated decreases sharply with increasing vaccine coverage, from 15 years when vaccine coverage is $50 \%$ or less to 4 years when vaccine coverage reaches $90 \%$ (figure 4b). This is because the reduction in absolute numbers of cervical cancer cases in Brazil achieved with increasing vaccination coverage leads to considerable savings in the costs of treating cervical cancer. As the budget remains constant, these savings then become available to be spent on decreasing the screening interval. In contrast, the screening interval remains high in the UK because the pre-vaccination number of cancer cases is low as organized screening has been in place for many years. The budget remains unchanged compared with the pre-vaccination budget throughout in both countries.

Figure 5 shows the effect of varying the screening coverage rate. In both countries, a substantial reduction in cervical cancer cases from the prevaccination level is obtained with vaccination alone (screening coverage $0 \%$ ). In the UK, the reduction is $20 \%$ and in Brazil, $43 \%$ (figure 5c). This also results in a budget reduction from prevaccination levels of $14 \%$ in Brazil and $30 \%$ in the UK (figure 5d). When screening is introduced at a low level of coverage, the screening interval is 


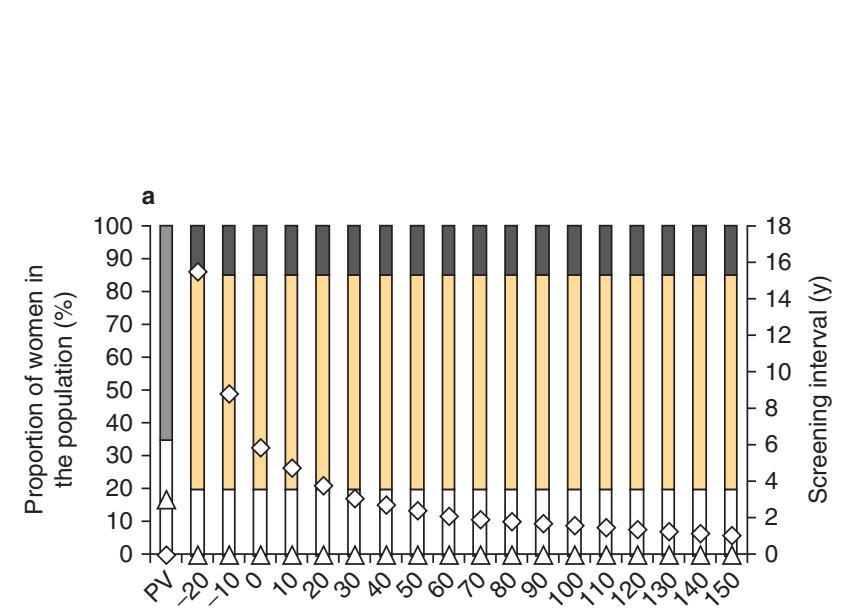

$\square$ Vaccination alone

Vaccination and screening

$\square$ Screening alone

$\square$ No prevention strategy

$\diamond$ Screening interval vaccinated

$\Delta$ Screening interval unvaccinated
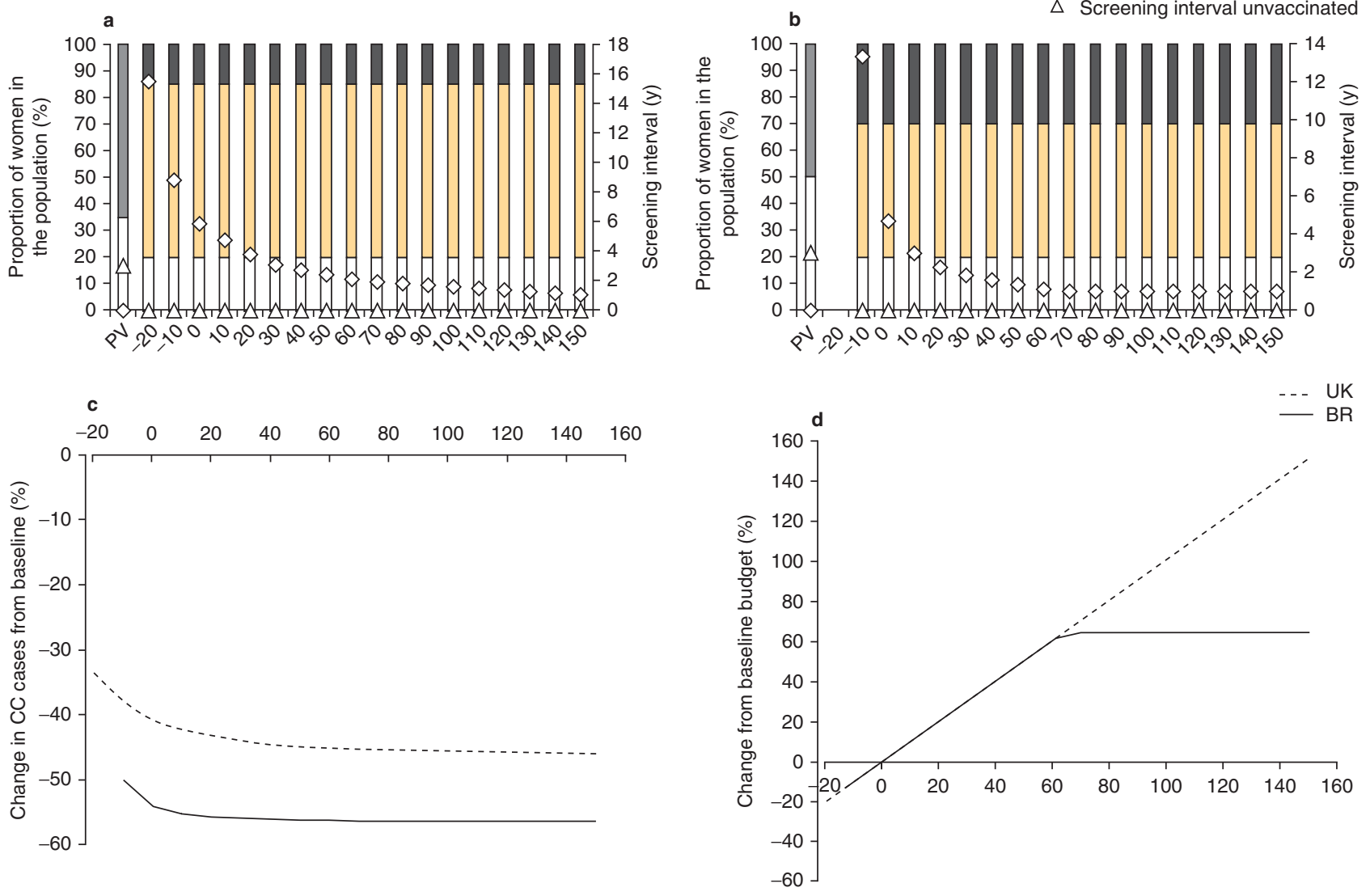

Change from baseline budget constraint (\%)

Fig. 3. Sensitivity analysis on budget constraint (all other constraints remaining at base-case value): (a) UK population distribution; (b) Brazil population distribution; (c) change from baseline in cervical cancer cases in both countries; and (d) change from baseline in budget in both countries. The left axes in (a) and (b) refer to the population distribution (bars), and the right to the screening interval $(\Delta$ : screened only; $\diamond:$ vaccinated and screened; where $\diamond$ or $\Delta=0$ correspond to having no women under that specific scenario, e.g. screened only or screened and vaccinated). $\mathbf{B R}=$ Brazil; $\mathbf{C C}=$ cervical cancer; $\mathbf{P V}=$ pre-vaccination strategy. 


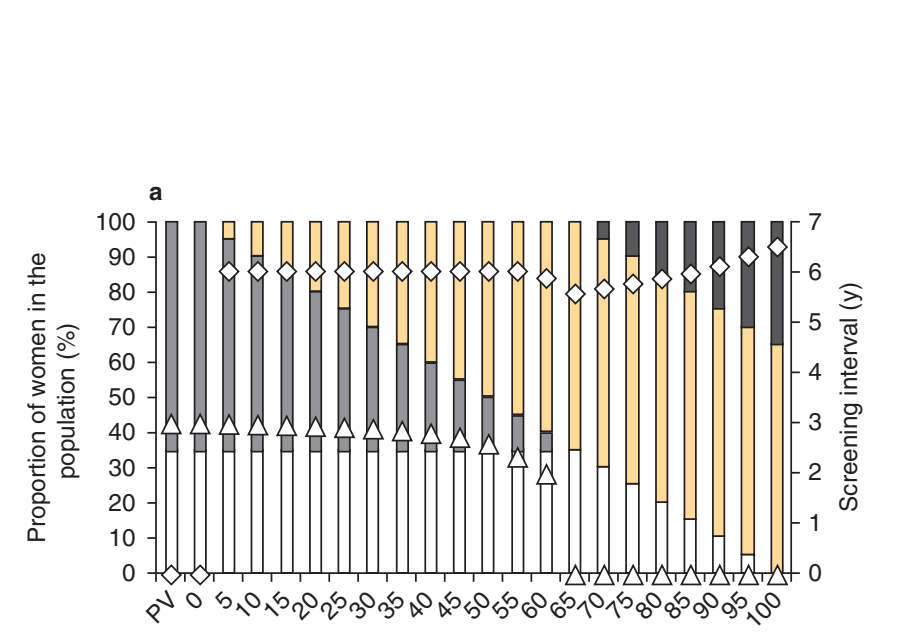

- - UK c

$10 \quad 20$

- BR

Vaccination and screening

$\square$ Screening alone

$\square$ No prevention strategy

$\Delta$ Screening interval unvaccinated

$\diamond$ Screening interval vaccinated

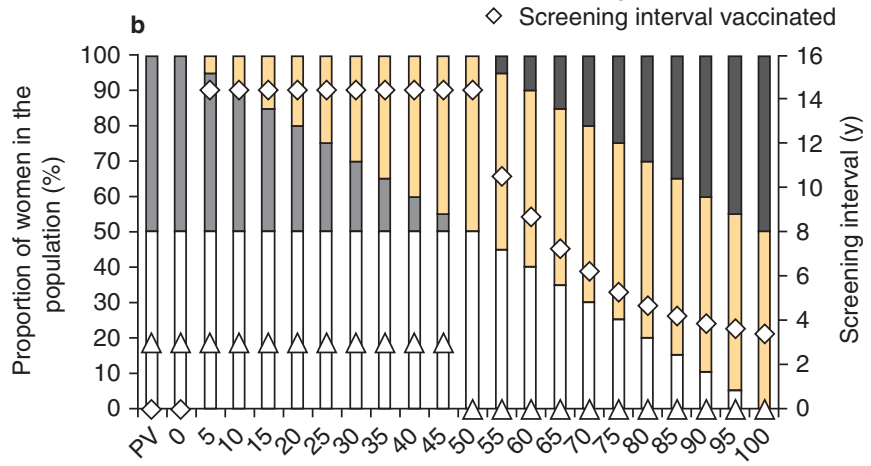

Vaccination coverage constraint (\%)

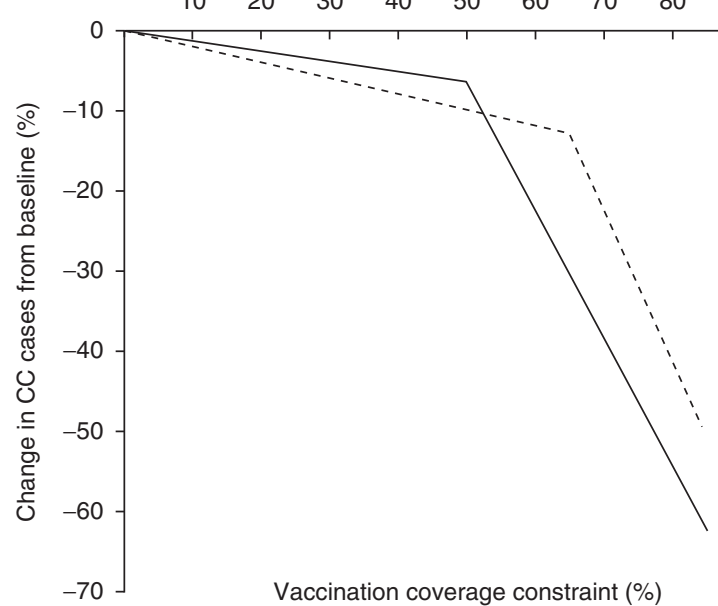

Fig. 4. Sensitivity analysis on vaccine coverage constraint (all other constraints remaining at base-case value): (a) UK population distribution; (b) Brazil population distribution; and (c) change from baseline in cervical cancer cases in both countries. The left axes in (a) and (b) refers to the population distribution (bars), and the right to the screening interval ( $\Delta$ : screened only; $\diamond:$ vaccinated and screened; where $\diamond$ or $\Delta=0$ correspond to having no women under that specific scenario, e.g. screened only or screened and vaccinated). $\mathbf{B R}=$ Brazil; $\mathbf{C C}=$ cervical cancer; $\mathbf{P V}=$ pre-vaccination strategy. 


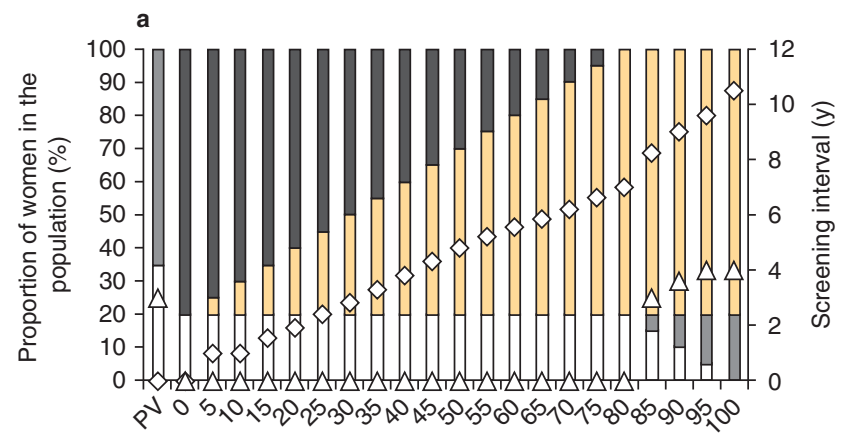

c

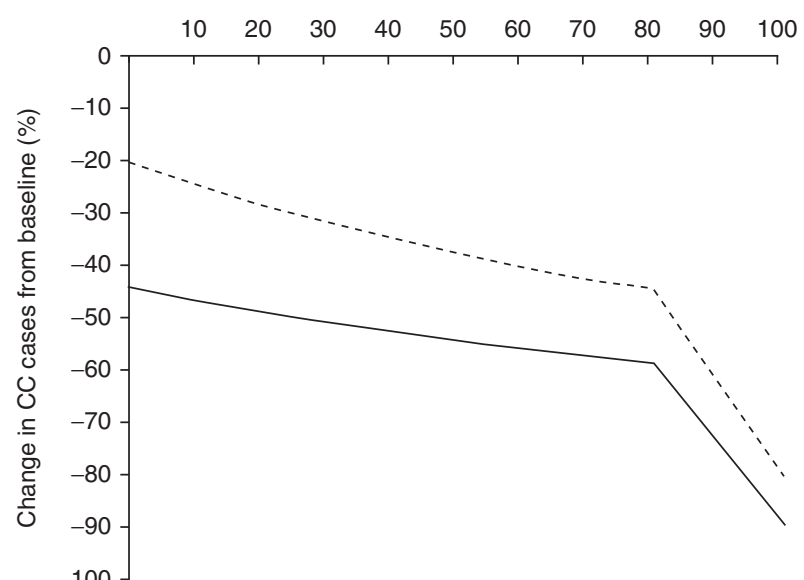

Vaccination alone

Vaccination and screening

Screening alone

No prevention strategy

$\Delta$ Screening interval unvaccinated

$\diamond$ Screening interval vaccinated

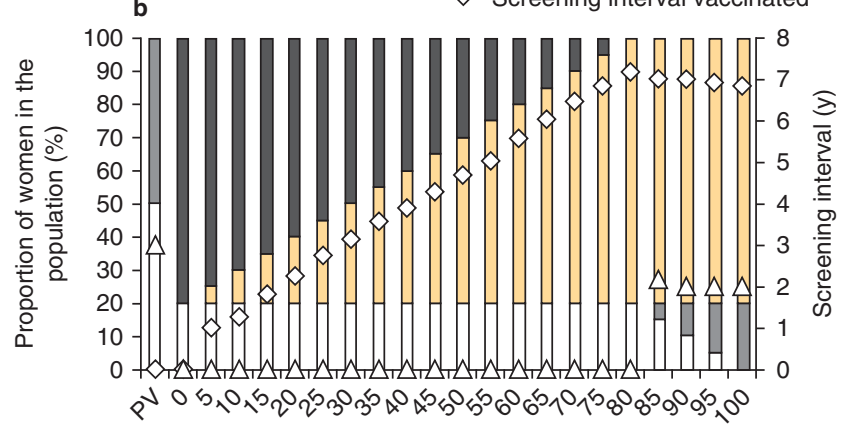

- UK
10
$30 \quad 40$
$50 \quad 60$
100

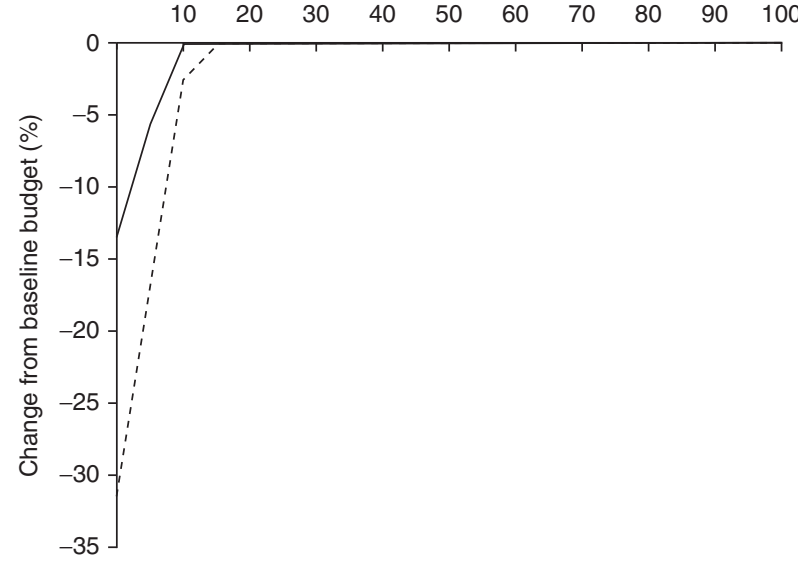

Screening coverage constraint (\%)

Fig. 5. Sensitivity analysis on screening coverage constraint (all other constraints remaining at base-case value): (a) UK population distribution; (b) Brazil population distribution (c) change from baseline in cervical cancer cases in both countries; and (d) change from baseline in budget in both countries. The left axis in (a) and (b) refers to the population distribution (bars), and the right to the screening interval ( $\Delta$ : screened only; $\diamond$ : vaccinated and screened; where $\diamond$ or $\Delta=0$ correspond to having no women under that specific scenario, e.g. screened only or screened and vaccinated). $\mathbf{B R}=$ Brazil; $\mathbf{C C}=$ cervical cancer; $\mathbf{P V}=$ pre-vaccination strategy. 

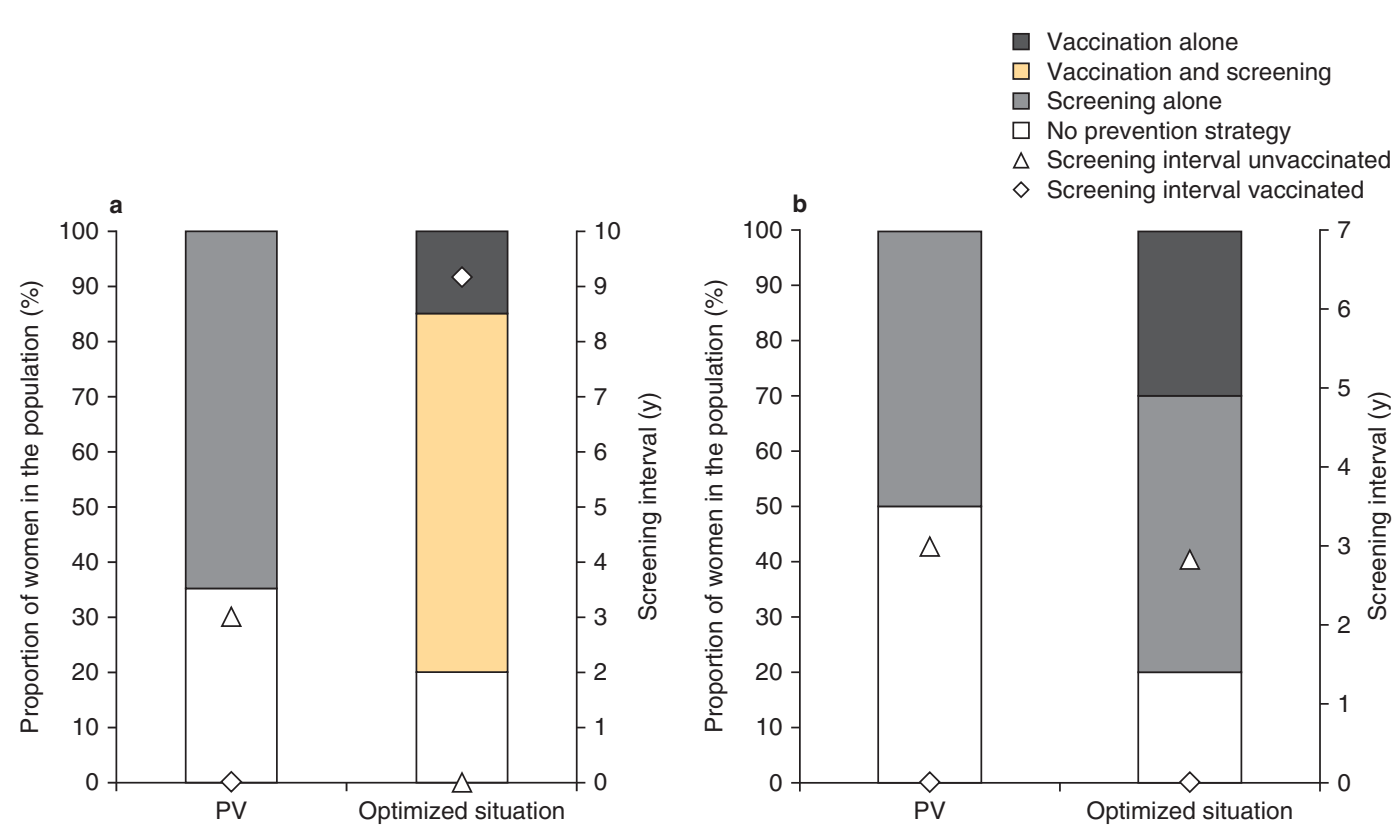

Fig. 6. Sensitivity analysis on vaccine duration of protection limited to 25 years (all other constraints remaining at base-case value) for (a) UK population distribution and (b) Brazil population distribution. The left axis refers to the population distribution (bars), and the right to the screening interval $(\Delta$ : screened only; $\diamond$ : vaccinated and screened; where $\diamond$ or $\Delta=0$ correspond to having no women under that specific scenario, e.g. screened only or screened and vaccinated). $\mathbf{P V}=$ pre-vaccination strategy.

initially short, steadily lengthening as screening coverage increases (figure $5 \mathrm{a}$ and $\mathrm{b}$ ). Clinical benefit improves steadily but marginally as screening coverage increases, with a sharp reduction in cervical cancer cases obtained only when the screening coverage rate becomes higher than the maximum vaccine coverage (figure $5 \mathrm{c}$ ).

Figure 6 shows the effect of reduced vaccine efficacy duration (25 years compared with lifetime) on the outcome. In both countries, the optimal mix of prevention strategies results in a reduction in cervical cancer compared with the prevaccination strategy. In the $\mathrm{UK}$, this reduction reached $26 \%$ associated with maximum screening and vaccination coverage and an increase in the screening interval to every 9 years. For Brazil, the maximum vaccination coverage rate is not reached and the optimization results in an extension of the population covered by any prevention strategy with screening alone at $50 \%$ and vaccination alone at $30 \%$ coverage, resulting in a reduction of cervical cancer by $35 \%$ compared with the pre-vaccination strategy.

\section{Discussion}

We developed an optimization program, using an evaluation model and an optimization model with linear programming, to define the optimal mix of cervical cancer prevention strategies (screening and/or vaccination) with the objective of minimizing the number of cervical cancer cases under constraints of budget and screening and vaccine coverage. We applied the program in two countries, the UK and Brazil. The optimal mix of intervention strategies under specific constraints represents realistic scenarios in terms of budget and screeningand vaccination-coverage limitations corresponding with the current situations in each country.

\section{Main Findings}

In the base case (no increase from pre-vaccination budget; maximum vaccination coverage of $80 \%$; maximum screening coverage equalling prevaccination levels), the optimal mix of strategies in the UK was $65 \%$ vaccination plus screening 
with a screening interval of 6 years, and 15\% vaccination alone. In Brazil, the optimal mix was $50 \%$ vaccination plus screening with a screening interval of 5 years, and $30 \%$ vaccination alone. The introduction of vaccination was associated with an increase in the screening interval from the pre-vaccination interval of 3 years to 6 years in the UK and to 5 years in Brazil, while reducing cervical cancer cases relative to the pre-vaccination levels by $41 \%$ in the UK and 54\% in Brazil. In both countries, the optimal coverage rates for both preventive interventions are at the maximum levels permitted in the model. The sensitivity analyses performed provide valuable information on the effect of changes in health policy or logistic parameters, such as altering the budget or the achievable screening or vaccination coverage. For example, increasing the budget permits shortening the screening interval, but the effect on the reduction in cervical cancer cases is modest and tends to reach an early plateau (figure 3 ). Vaccination alone (screening coverage set to $0 \%$ ) could provide a reduction in cervical cancer cases compared with the prevaccination situation of screening alone with a lower budget (figure 5). If screening coverage is fixed at its maximum (the pre-vaccination coverage level), the addition of vaccination provides important added clinical benefit only if the vaccination coverage rate becomes higher than the screening coverage rate (see figure 4). This is because the second intervention is initially applied to the proportion of the population who also received the first intervention. So, only when the coverage of the second intervention exceeds the first does it begin to cover that part of the population not covered by the first. For example, when screening coverage is set at $50 \%$ and vaccination at $40 \%$, the $40 \%$ receiving vaccination are assumed to also receive screening. So the actual mix is $40 \%$ vaccination plus screening, $10 \%$ screening alone and $50 \%$ no prevention. When screening coverage is set at $50 \%$ and vaccination coverage reaches $60 \%$, the mix is $50 \%$ screening plus vaccination, $10 \%$ vaccination alone and $40 \%$ no prevention.

The analyses also indicate that in the absence of any prevention against cervical cancer, it would economically be more favourable to invest in vaccination first and subsequently in screening (vacci- nation alone prevents a higher number of cervical cancer cases compared with the pre-vaccination situation of cytology-based screening alone). The combination of vaccination and cytology-based screening under the pre-vaccination budget constraint always leads to an improved outcome compared with the pre-vaccination level. The maximum benefit is seen when both interventions are additive, i.e. when the coverage of the second exceeds the coverage of the first (see figures 4 and 5). Meanwhile the additive effect achieved differs between countries. Adding vaccination to screening (figure 4) or screening to vaccination (figure 5) has a greater effect on cervical cancer reduction in Brazil than in the UK. This discrepancy reflects the combination of higher screening frequency with higher vaccine coverage rates in Brazil than in the UK because the screening cost in Brazil is low.

\section{Strengths of the Optimization Analysis}

The key strength of optimization modelling is its ability to evaluate multiple combinations of different interventions and identify the mix that provides the maximum expected health benefit (reduction in cervical cancer cases) at the minimum expected cost. In addition, it allows the decision maker to set constraints reflecting local conditions, such as a limited available budget or limited achievable coverage rates.

Optimization modelling provides valuable additional information compared with either costeffectiveness or budget impact analysis, since it explicitly evaluates multiple available options to select that combination that fulfils all the constraints introduced in the model while obtaining the most efficient result: lowest cost for the best outcome. ${ }^{[31-37]}$ This is especially suited for assessing public health interventions, where large but limited budgets must be allocated between different intervention options to allow a specific health goal to be reached. Compared with cost-effectiveness analysis for decision making, optimization modelling does not require a pre-specified cost-effectiveness threshold, associated with much debate in the literature. To our knowledge, this is the first published study of optimization modelling applied to the health policy problem of selecting the best 
mix of interventions in the prevention of cervical cancer.

Meanwhile, if the decision problem concerns only a few alternative interventions, it may be more straightforward to use standard cost-effectiveness evaluation, supplemented with budget impact analysis to assess the relative affordability of the competing interventions. When comparing the results from a cost-effectiveness analysis with that of an optimization model, both should obtain the same end result where cost-effectiveness analysis is aiming for the lowest incremental cost-effectiveness ratio (ICER). However, from a practical point of view, optimization models may provide a greater range of answers that are easier to understand, and may better address questions of affordability under realistic budget constraints.

\section{Limitations of the Optimization Analysis}

Optimization modelling also has its limitations. The model outcome is obtained when an optimal mix of different prevention strategies is applied and sustained over a lifetime. Many years will elapse before reaching that state. The results therefore represent an optimal 'ideal' situation that provides a direction to be followed but not a condition that can easily be reached tomorrow.

A static Markov model is used to estimate the cost and outcomes of each potential strategy at steady state across a population over a 1-year period. As the optimization model takes results from the evaluation model, the robustness of the optimization results therefore also depend upon the validity of the evaluation model. The latter does not account for dynamic effects of vaccination on infection, such as herd protection. It also assumes stability in the population demographic structure over time. Using a dynamic model as the evaluation model instead of the static model would however be much more cumbersome to adapt to countries with limited data. It would also lead to non-linearity between coverage rates and the outcomes, and thus be difficult to include in the optimization model.

No discount was applied as the model presents a steady state evaluation over a 1-year period. Given the time lag needed to reach steady state, discounting could be considered, but the means of implementation is currently unclear.

The models presented here are deterministic: costs and effects in all combinations are known. It is possible to characterize the allocation problem under conditions of uncertainty by reformulating the linear programming problem as a stochastic analysis. However, existing approaches for such stochastic analyses are limited. ${ }^{[46]}$ It is an area of future research, although the principal outcome of evaluating uncertainty is to indicate the need and the potential value of searching for more detailed and specific information. ${ }^{[46]}$

The results are dependent upon the strategies initially investigated with the evaluation model. Therefore, including a vaccine with a different profile or a screening programme with different characteristics (HPV testing instead of cytology-based screening) may lead to other optimal scenarios. Limitations (data availability, time, computing power, appropriate models, ...), however, restrict the number of scenarios that can be included. Furthermore, the analysis does not take into account the possibility that the introduction of vaccination may affect the sensitivity of the screening test. ${ }^{[47,48]}$ Additional research would be needed to explore alternative screening scenarios.

Our model assumes that the interventions evaluated are completely divisible in terms of intervals between screening tests and coverage. It ignores possible fixed set-up and implementation costs associated with interventions, which could constrain their divisibility. The fixed costs could be large for screening coverage, such as setting up the necessary infrastructure to collect and analyse samples, or implementing health education programmes to increase awareness and overcome psychological and cultural barriers to uptake. Conversely, they may be relatively low for vaccine implementation in situations where other vaccination programmes in the relevant age group are already established. In addition, the incremental implementation cost by coverage is unlikely to be linear. Disregarding such indivisible or non-linear costs could be important in practice.

Another issue is that this approach only partially addresses equity problems. The optimization model considers the whole female population as 
homogeneous, except when the upper bound on the population without prevention constraint becomes active. This constraint implies that, when the maximum coverage rate is reached for both screening and vaccination, corresponding therefore to the target coverage set by the decision makers, if a woman is not screened, she is also likely not to be vaccinated. This constraint, however, does not apply when maximal coverage is not reached for both screening and vaccination, as observed in Brazil with the sensitivity analysis on the duration of protection. In this case, the optimization model tends to maximize the prevention coverage rate by identifying separately a proportion with screening alone and a proportion with vaccination alone, a situation that is unlikely to occur in a real-life setting. If such a group could easily be identified being exposed to any prevention strategy, by making particular efforts to improve the uptake of a prevention strategy amongst them, it is likely to be a good health policy decision. Optimization models may therefore be helpful to determine the optimal mix of interventions to which a group will best adhere. Furthermore, the model can be adapted to take into account regional disparities, such as differences in constraints, epidemiology and costs, which could be substantial in large countries with diverse regional health settings. In such settings, the application of regional optimization models allowing for these disparities might provide more useful information (than a single ICER or budget impact analysis) to the local decision maker than country-level evaluation.

\section{Conclusion}

The optimization model presented here directly addresses the information needed to inform health policy decisions on the allocation of limited resources to prevent cervical cancer in order to achieve the best possible outcome.

\section{Acknowledgements}

BS and ND designed the study; BS, ND and TB researched and agreed on data input into the model and the variables to be tested. ND designed the computer model and conducted the simulations and analysis. Data analysis was conducted by
ND under the supervision of BS and TB. ND and BS drafted the manuscript. All authors commented on drafts and contributed to the final version. BS is the guarantor of the study.

Medical writing assistance was provided by Carole Nadin and Niels Neymark and publication coordination by Laure Delbecque and Maud Boyer (Business and Decision) on behalf of GlaxoSmithKline Biologicals, Belgium.

This study was funded by GlaxoSmithKline Biologicals, Belgium.

All authors are employees of GlaxoSmithKline Biologicals, Wavre, Belgium and have stock options (GlaxoSmithKline). They have no other relationships/conditions/circumstances that present a potential conflict of interest.

The authors would like to very much thank the valuable comments of the anonymous referees on the initial submission of the manuscript, as well as Christophe Sauboin from GlaxoSmithKline Biologicals, Belgium for his contribution in defining the mathematical formulations of the optimization model.

\section{References}

1. Cancer Research UK. Cervical cancer - UK incidence statistics [online]. Available from URL: http://info.cancer researchuk.org/cancerstats/types/cervix/incidence/index.htm [Accessed 2010 Jul 26]

2. Ferlay J, Shin HR, Bray F, et al. GLOBOCAN 2008: cancer incidence, mortality and prevalence worldwide in 2008 [online]. Available from URL: http://globocan.iarc.fr [Accessed 2010 Jun 22]

3. Antilla A, Aoki D, Arbyn M, et al. Cervix cancer screening. Lyon: IARC Press, 2005. Report no.: 10

4. Arbyn M, Raifu AO, Autier P, et al. Burden of cervical cancer in Europe: estimates for 2004. Ann Oncol 2007 Apr 10; 18 (10): 1708-15

5. Denny L, Quinn M, Sankaranarayanan R. Chapter 8: screening for cervical cancer in developing countries. Vaccine 2006 Aug 31; 24 Suppl. 3: S3/71-7

6. Walboomers JM, Jacobs MV, Manos MM, et al. Human papillomavirus is a necessary cause of invasive cervical cancer worldwide. J Pathol 1999; 189 (1): 12-9

7. zur Hausen $H$. Intracellular surveillance of persisting viral infections: human genital cancer results from deficient cellular control of papillomavirus gene expression. Lancet 1986 Aug 30; 328 (8505): 489-91

8. Smith JS, Lindsay L, Hoots B, et al. Human papillomavirus type distribution in invasive cervical cancer and high-grade cervical lesions: a meta-analysis update. Int J Cancer 2007 Apr 2; 121 (3): 621-32

9. Munoz N, Castellsague X, de Gonzalez AB, et al. HPV in the etiology of human cancer. Vaccine 2006 Jun 23; 24 Suppl. 3: S1-10

10. Paavonen J, Naud P, Salmerón J, et al. Efficacy of human papillomavirus (HPV)-16/18 AS04-adjuvanted vaccine against cervical infection and precancer caused by oncogenic HPV types (PATRICIA): final analysis of a doubleblind, randomised study in young women. Lancet $2009 \mathrm{Jul}$ 25; 374 (9686): 301-14

11. Villa LL, Costa RL, Petta CA, et al. High sustained efficacy of a prophylactic quadrivalent human papillomavirus 
types 6/11/16/18 L1 virus-like particle vaccine through 5 years of follow-up. Br J Cancer 2006 Dec 4; 95 (11): 1459-66

12. FUTURE II Study Group. Quadrivalent vaccine against human papillomavirus to prevent high-grade cervical lesions. N Engl J Med 2007 May 10; 356 (19): 1915-27

13. Garland SM, Hernandez-Avila M, Wheeler CM, et al. Quadrivalent vaccine against human papillomavirus to prevent anogenital diseases. N Engl J Med 2007 May 10; 356 (19): 1928-43

14. Harper DM, Franco EL, Wheeler C, et al. Efficacy of a bivalent $\mathrm{L} 1$ virus-like particle vaccine in prevention of infection with human papillomavirus types 16 and 18 in young women: a randomised controlled trial. Lancet 2004 Nov 13; 364 (9447): 1757-65

15. Munoz N, Kjaer SK, Sigurdsson K, et al. Impact of human papillomavirus (HPV)-6/11/16/18 vaccine on all HPVassociated genital diseases in young women. J Natl Cancer Inst 2010 Feb 5; 102 (5): 325-39

16. Szarewski A. HPV vaccine: cervarix. Expert Opin Biol Ther 2010 Mar; 10 (3): 477-87

17. Skinner R, Apter D, Chow SN, et al. Cross-protection efficacy of Cervarix ${ }^{\mathrm{TM}}$ against oncogenic HPV types beyond HPV-16/18 [abstract no. O-29.01] 25th International Papillomavirus Conference; 2009 May 8-14; Malmö

18. Brown DR, Kjaer SK, Sigurdsson K, et al. The impact of quadrivalent human papillomavirus (HPV; types 6,11, 16, and 18) L1 virus-like particle vaccine on infection and disease due to oncogenic nonvaccine HPV types in generally HPV-naive women aged 16-26 years. J Infect Dis 2009 Apr 1; 199 (7): 926-35

19. Brabin L, Roberts SA, Stretch R, et al. Uptake of first two doses of human papillomavirus vaccine by adolescent schoolgirls in Manchester: prospective cohort study. BMJ 2008; 336 (7652): 1056-8

20. Stokley S, Dorell C. National, state, and local area vaccination coverage among adolescents aged 13-17 years United States, 2008. MMWR Morb Mortal Wkly Rep 2010 Sep 18; 58 (36): 997-1001

21. Wong C, Berkowitz Z, Wai Lee J, et al. National HPV vaccine uptake and vaccination predictors and barriers for girls 8-17 years old - United States, 2008. National STD Prevention Conference; 2010 Mar 8-11; Atlanta (GA) [online]. Available from URL: http://cdc.confex.com/cdc/std 2010/webprogram/Paper22960.html [Accessed 2010 Jul 19]

22. Brisson M, Van de Velde N, Boily MC. Economic evaluation of human papillomavirus vaccination in developed countries. Public Health Genomics 2009; 12 (5-6): 343-51

23. Kim JJ, Brisson M, Edmunds WJ, et al. Modeling cervical cancer prevention in developed countries. Vaccine 2008 Aug 19; 26 Suppl. 10: K76-86

24. Marra F, Cloutier K, Oteng B, et al. Effectiveness and cost effectiveness of human papillomavirus vaccine: a systematic review. Pharmacoeconomics 2009; 27 (2): 127-47

25. Suárez E, Smith JS, Bosch FX, et al. Cost-effectiveness of vaccination against cervical cancer: a multi-regional analysis assessing the impact of vaccine characteristics and alternative vaccination scenarios. Vaccine 2008 Sep 15; 26 Suppl. 5: F29-45

26. Adang E, Voordijk L, Jan van der Witt G, et al. Costeffectiveness analysis in relation to budgetary constraints and reallocative restrictions. Health Policy 2005 Oct; 74 (2): 146-56

27. Claxton K. The irrelevance of inference: a decision-making approach to the stochastic evaluation of health care technologies. J Health Econ 1999 Jun; 18 (3): 341-64

28. Gafni A, Birch S. Incremental cost-effectiveness ratios (ICERs): the silence of the lambda. Soc Sci Med 2006 May; 62 (9): 2091-100

29. Hutubessy RC, Bendib LM, Evans DB. Critical issues in the economic evaluation of interventions against communicable diseases. Acta Trop 2001 Mar 30; 78 (3): 191-206

30. World Health Organization. Making choices in health: WHO guide to cost-effectiveness analysis. Geneva: WHO, 2003

31. Drummond MF. Principles of economic appraisal in health care. Oxford: Oxford University Press, 1980

32. Earnshaw SR, Richter A, Sorensen SW, et al. Optimal allocation of resources across four interventions for type 2 diabetes. Med Decis Making 2002 Sep; 22 (5 Suppl.): S80-91

33. Epstein DM, Chalabi Z, Claxton K, et al. Efficiency, equity, and budgetary policies: informing decisions using mathematical programming. Med Decis Making 2007 Mar; 27 (2): 128-37

34. Stinnett AA, Paltiel AD. Mathematical programming for the efficient allocation of health care resources. J Health Econ 1996 Oct; 15 (5): 641-53

35. Earnshaw SR, Hicks K, Richter A, et al. A linear programming model for allocating HIV prevention funds with state agencies: a pilot study. Health Care Manag Sci 2007 Sep; 10 (3): $239-52$

36. Richter A, Hicks KA, Earnshaw SR, et al. Allocating HIV prevention resources: a tool for state and local decision making. Health Policy 2008 Sep; 87 (3): 342-9

37. Weniger BG, Chen RT, Jacobson SH, et al. Addressing the challenges to immunization practice with an economic algorithm for vaccine selection. Vaccine 1998 Nov; 16 (19): 1885-97

38. Earnshaw SR, Dennett SL. Integer/linear mathematical programming models: a tool for allocating healthcare resources. Pharmacoeconomics 2003; 21 (12): 839-51

39. Luenberger DG, Yinyu Y. Linear and nonlinear programming. 3rd ed. New York: Springer, 2008

40. Debicki D, Ferko N, Demarteau N, et al. Comparison of detailed and succinct cohort modelling approaches in a multi-regional evaluation of cervical cancer vaccination. Vaccine 2008 Sep 15; 26 Suppl. 5: F16-28

41. Colantonio L, Gómez JA, Demarteau N, et al. Costeffectiveness analysis of a cervical cancer vaccine in five Latin American countries. Vaccine 2009; 27 (40): 5519-29

42. Castellsague X, de Sanjose S, Aguado T, et al. HPV and cervical cancer in the world: 2007 report. Vaccine 2007; 25 Suppl. 3: C1-230

43. Tjalma W, Paavonen J, Naud P, et al. Efficacy of the HPV16/18 AS04-adjuvanted vaccine against abnormal cytology and low-grade histopathological lesions in an oncogenic HPV-naïve population [abstract no. A-171-0004-01446]. 16th International Meeting of the European Society for Gynaecological Oncology (ESGO); 2009 Oct 11-14 Oct; Belgrade

44. Department of Health. Cervical screening programme, England: 2003-2004 [online]. Available from URL: http:// www.dh.gov.uk/assetRoot/04/09/63/75/04096375.pdf [Accessed $2010 \mathrm{Jul} 1$ ] 
45. David MP, Van Herck K, Hardt K, et al. Long-term persistence of anti-HPV-16 and -18 antibodies induced by vaccination with the AS04-adjuvanted cervical cancer vaccine: modeling of sustained antibody responses. Gynecol Oncol 2009 Feb; 115 (3 Suppl. 1): S1-6

46. McKenna C, Chalabi Z, Epstein D, et al. Budgetary policies and available actions: a generalisation of decision rules for allocation and research decisions. J Health Econ 2010 Jan; 29 (1): 170-81

47. Franco EL, Cuzick J. Cervical cancer screening following prophylactic human papillomavirus vaccination. Vaccine 2008 Mar 14; 26 Suppl. 1: A16-23
48. Rogoza RM, Ferko N, Bentley J, et al. Optimization of primary and secondary cervical cancer prevention strategies in an era of cervical cancer vaccination: a multiregional health economic analysis. Vaccine 2008 Sep 15; 26 Suppl. 5: F46-58

Correspondence: Mrs Nadia Demarteau, Health Economics, Global Vaccine Development, GlaxoSmithKline Biologicals, Avenue Fleming 20, B-1300 Wavre, Belgium.

E-mail: nadia.x.demarteau@gskbio.com 